\title{
Evidence-based dentistry: let's talk about experimental evidence
}

\author{
Deborah Martin'
}

\section{Key points}

\begin{abstract}
As our reliance on empirical evidence within the dental community grows, now could be the time to understand why the experiment methodology is being favoured. This article reviews the background literature in greater detail, relating to what empirical evidence is and its relevance to evidence-based dentistry (EBD). It explores why empirical evidence is becoming synonymous with the outcome of an experiment methodology only. Furthermore, the article discusses why these preferences may not be truly justified and looks at the implications of the findings for the future of EBD.
\end{abstract}

\section{Introduction}

Concato (2004) wrote that experimental evidence is given too much authority over the other types of empirical evidence collected in medicine. ${ }^{1}$ Evidence-based dentistry (EBD) gives different levels of importance to the methodologies used to collect the evidence, and there is a current preference towards experimentally-collected data, usually collected by quantitative means versus observational data typically obtained via qualitative methods. ${ }^{2}$ As our reliance on empirical evidence within the dental community grows, now could be the time to understand why the experiment methodology is being favoured and if insights are being missed because certain types of evidence are being ignored.

This is relevant to EBD because not all questions relevant to general dental practice can be answered with the experiment method, due to certain ethical and practical reasons. The article is structured as follows: first, I summarise the background literature in greater detail relating to what empirical

${ }^{1}$ Cripps Dental Centre, Cripps Hill, Nottingham, UK. Correspondence to: Deborah Martin

Email: Deborah@aeglenetwork.co.uk

Refereed Paper.

Accepted 21 November 2018

DOI:10.1038/s41415-019-0201-2 evidence is and its relevance to EBD; secondly, I explore why empirical evidence is becoming synonymous with the outcome of an experiment methodology only; thirdly, I comment on why these preferences may not be truly justified; and lastly the paper discusses the implications of the findings for the future of EBD.

\section{Empirical evidence}

Let's begin with exploring the term 'empirical evidence.' 'Empirical' means based on observation or experiment methods; 'evidence' relates to the actual facts or data collected. ${ }^{3}$ In brief, any data observed directly or through experimentation are empirical evidence. ${ }^{4}$ In 1972, Cochrane published a book suggesting that patient care should be based on objective evidence and not the beliefs of the doctor; this may have started the unjustified preference towards the experiment due to a misunderstanding surrounding research methodologies in evidence-based medicine (EBM). ${ }^{5}$

Accordingly, observational data are not related to the beliefs of the doctor but are the methodological collection method conducted via qualitative methods, and experimental data are usually collected via quantitative methods. Importantly, the research question determines which data method is selected to collect the most suitable data to answer the question. It follows that, the social science approach of 'concept questions' favours the observation, and the science methodologies favour the hypothesis, and neither approach relies on the 'beliefs' of the doctor, making each method valid.

That said, pure qualitative research is underutilised in EBD and is a valid scientific method based on observation data, which gathers non-numerical data. This type of research data refers to meanings, concepts and descriptions and answers how, why and when a certain phenomenon occurs, rather than the quantitative approach, which measures and tests a hypothesis. ${ }^{6}$

\section{The qualitative approach}

In EBD, the social science approach of collecting qualitative data would be the case study observation. In the past, case studies tried to answer a hypothesis question, which resulted in a weak conclusion. The case study is the weakest methodology to answer the hypothesis question and, thus, why the hierarchy of evidence may be choosing to place the case study last. But in fact, qualitative and quantitative research are both equally valid scientific approaches to collecting empirical evidence, and both are relevant to evidencebased practice. 
However, the strength of qualitative data collection is being overlooked in current evidence-based practice (EBP), despite the fact that both scientific methods are valid. Consequently, it is the type of question which determines the method selected and the scientific hypothesis is taking precedence, but not all questions can be answered via an experiment. As Einstein once said, 'Not everything that is important can be measured, and not everything that can be measured is important'.

One example of qualitative data being overlooked is through analysis of current dental journals. There are few pure case study articles, especially detailing the useful grounded theory approach. This approach allows a theory to develop from the observational data. Currently, qualitative data is presented as an answer to the concept question but via questionnaires etc. This type of methodology is not as strong as the case pure study for doing this. Furthermore, knowledge may be enhanced with the addition of research skills associated with social science balanced with the science methodologies, which are both needed to support and understand EBD.

\section{The hierarchy of evidence}

To illustrate, empirical evidence is becoming synonymous with only the outcome of experiments due to a misunderstanding surrounding the hierarchy of evidence. ${ }^{7}$ The current hierarchy is correct in relation to the testing of a hypothesis and quantitative data, but not if answering a different type of question. Urgent revision may be needed to support the hierarchy in reverse for questions that require qualitative data and comment on concepts that are not suitable for the experimental process. So, this preference may not be justified as each scientific method is as valid as the other, but just at collecting different data sets and answering different questions.

Borgerson (2009) states that the justification for the hierarchy is not absolute, but they are unable to give details on the reasoning for this. However, this article may go some way in explaining why this is happening. ${ }^{8}$ This is because the hypothesis is increasingly preferred as the profession becomes more science-based. Both scientific methods are as valid as each other but are used to investigate different questions. Therefore, valuable insights from the social-sciences perspective are being overlooked in favour of its cousin, the experiment. to the detriment of medicine. Insights from qualitative data relating to phenomenon may be being missed because of poor understanding of the different research methodologies.

\section{Conclusion}

Currently, certain forms of methodologies used to collect evidence seem to be given preference over other forms of data in evidence-based practice. This article explored what evidence is and why certain types of data are being preferred over others. It argued why those preferences are not truly justified, as insights are being missed because certain types of evidence are being ignored.
In brief, my observations seem to be paradigmatically opposed to the dominant opinion in my area, but should this continue? The systematic review or randomised control trial seems superior for answering the hypothesis, but the case study seems superior for answering the 'concept question'. As a dentist with a social-science master's degree, paid for by Health Education England, I believe it is now time that our social science scientific methodology is embraced and recognised as equal to the science methodology in EBD. Mainly, because the evidence suggests that it actually is.

Interestingly, the questions asked in general practice are ideally suited to the social science approach. In the future, is grounded theory going to rival the randomised control trial for research conducted in general practice, and will the profession start to adopt it?

\section{References}

1. Concato J. Observational versus experimental studies: What's the evidence for a hierarchy? NeuroRx 2004; 1: 341-347.

2. Greenhalgh T. How to read a paper: getting your bearings (deciding what the paper is about). BMJ 1997: 315: 243-246.

3. Merriam-Webster. Merriam-Webster's Collegiate Dictionary. 11th ed. Springfield, MA: Merriam-Webster Inc., 2009.

4. Kothari C R. Research Methodology: Methods and Techniques. 3rd ed. Delhi: New Age International, 2012.

5. Cochrane A L. Effectiveness and efficiency: Random reflections on health services. London: Nuffield Trust, 1972.

6. Audi R (ed). The Cambridge Dictionary of Philosophy. 3rd ed. Cambridge: Cambridge University Press, 2015.

7. Stegenga J. Down with the hierarchies. Topoi 2014; 33: 313-322.

8. Borgerson K. Valuing evidence: bias and the evidence hierarchy of evidence-based medicine. Perspectives Biol Med 2009; 52: 218-233. 